\title{
Scar endometriosis: an uncommon surgical aftermath
}

\author{
Sumedha Pathade*, Shruti Panchbudhe, Prasad Deshmukh, Arun Nayak
}

Department Obstetrics and Gynaecology, Lokmanya Tilak Municipal Medical College, Mumbai, Maharashtra, India

Received: 31 December 2021

Accepted: 09 February 2022

\section{*Correspondence:}

Dr. Sumedha Pathade,

E-mail: pathadesumedha@gmail.com

Copyright: ( $)$ the author(s), publisher and licensee Medip Academy. This is an open-access article distributed under the terms of the Creative Commons Attribution Non-Commercial License, which permits unrestricted non-commercial use, distribution, and reproduction in any medium, provided the original work is properly cited.

\begin{abstract}
Endometriosis is a benign condition wherein endometrial tissue lies outside the uterine cavity. Abdominal scar endometriosis is a rare entity following pelvic surgeries caused as a result of deposition of endometrial tissue at scar site. Patient usually presents with triad of presence of mass over scar site, cyclical pain during menses and bleeding. Histopathological examination remains gold standard for diagnosis in which the presence of endometrial glands, stroma and hemosiderin laden macrophages can be seen. We presented a case of 35 years old lady with history of caesarean section done 3 years back who came with complaints of swelling over the scar site and cyclical pain with tenderness present over lump. Ultrasonography revealed presence of firm mass which was removed by wide local excision and histopathology confirmed the diagnosis of scar endometriosis.
\end{abstract}

Keywords: Scar endometriosis, LSCS, Rectus sheath

\section{INTRODUCTION}

Endometriosis is defined as deposition of endometrial tissue anywhere other than uterine cavity. It occurs in 5$10 \%$ of all women often resulting in debilitating pain and infertility. ${ }^{1}$ It can be extra pelvic or endopelvic. The most common site of extra pelvic deposition is scar endometriosis which is rare entity. Majority of scar endometriosis have been reported after obstetrical or gynaecological procedures such as caesarean delivery, hysterotomy, hysterectomy, episiotomy and tubal ligations. ${ }^{2}$ The reported incidence of scar endometriosis after caesarean section is $0.3-0.4 \%$ and following hysterotomy is $1-2 \% .^{3}$ Caesarean section scar is the most common surgical site of abdominal scar endometriosis. The lesions of scar endometrioma have been confused with lipoma, hematoma, stitch granulomas, incisional hernia, cyst, abscess.

\section{CASE REPORT}

A 35-year-old patient, para 3 living 3 death 1 presented to gynecological outpatient department with complaints of presence of lump over the left lateral edge of previous pfannenstiel scar. She underwent caesarean section 3 years ago and 6 months post caesarean section she started developing a lump over the left side of the scar which gradually increased over a period of two and a half years to reach the present size of $4 \times 3 \times 2 \mathrm{~cm}$ (Figure 1 ). She gave history of cyclical pain during menses at the scar site however she did not report any symptoms of dysmenorrhea, dyspareunia or chronic pelvic pain. Her medical and surgical history were not significant.

On general examination all her vital parameters were normal. On per abdomen examination there was $4 \times 3 \mathrm{~cm}$ nodular lump over left lateral edge of caesarean scar site which had blackish discoloration with punctate black spots. The lump was tender, non-mobile, firm in consistency. Per speculum and per vaginal examination were normal. All her routine blood investigations were normal. Ultrasonography of the abdomen revealed a $4.2 \times 2 \times 3.24 \mathrm{~cm}$ mass which was heterogenous, hypoechoic lesion with mild internal vascularity at caesarean section scar site in subcutaneous plane in left lumbar region. No intralesional calcifications were seen (Figure 2). 
She was posted for surgical excision of the scar endometrioma. An elliptical incision was taken with a margin of $1 \mathrm{~cm}$ was taken at scar endometrioma site (Figure 3 ). It was deepened to reach till the base of endometrioma which was seen adherent to the rectus sheath leading to inadvertent opening of the rectus sheath to achieve enmass removal of endometrioma (Figure 4). However, no extension was seen in rectus muscle layer. The defect in the rectus sheath was reenforce with non-absorbable suture (ethilon 1) and rest of the layers of the skin closed in layers. The scar endometrioma was removed en-mass and sent for histopathological examination (Figure 5). Post-operative period was uneventful and suture removal was done on day 10. She followed up after 6 weeks and 12 weeks with no complaints. Histopathological examination revealed tissue showing multiple endometrial glands surrounded by endometrial stroma consistent with features suggestive of scar endometriosis (Figure 6).

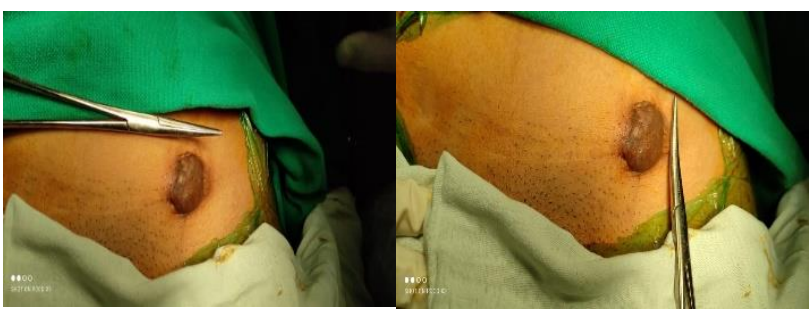

Figure 1: 4×3 cm nodular lump over left lateral edge of caesarean scar site.

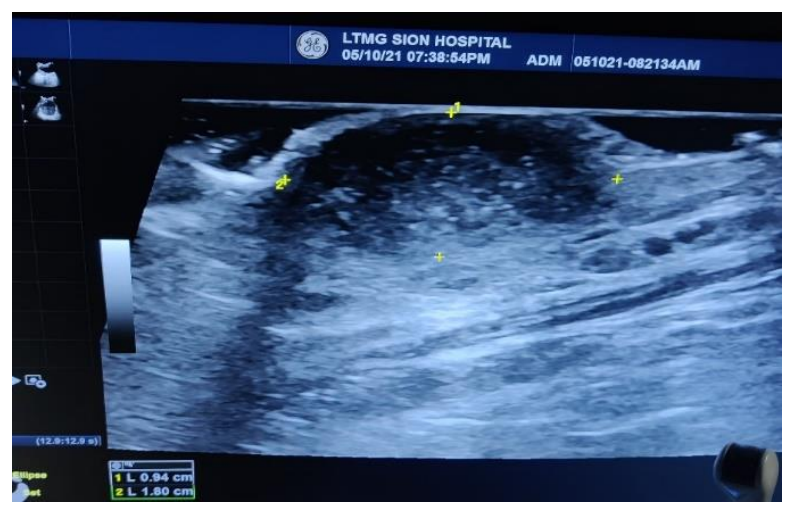

Figure 2: Ultrasonographic picture of scar endometrioma.

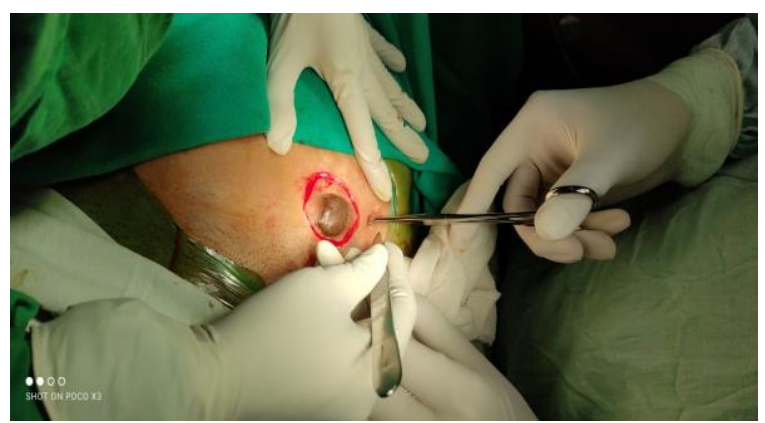

Figure 3: Elliptical incision with a margin of $1 \mathrm{~cm}$ at scar endometrioma site.

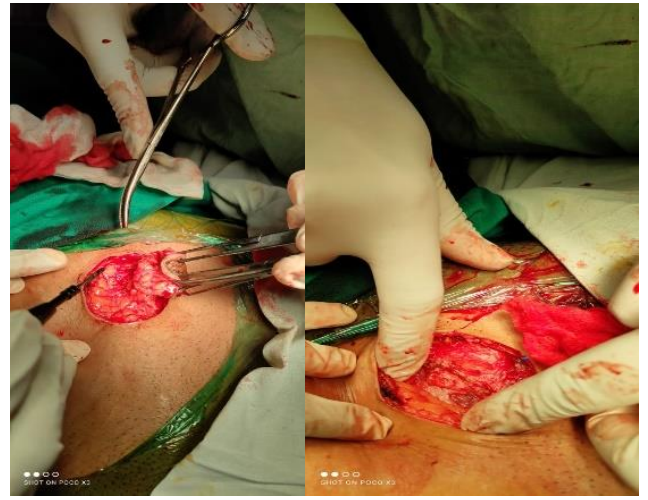

Figure 4: Scar endometrioma excised with elliptical incision with extension till rectus sheath causing defect in rectus sheath after its excision.

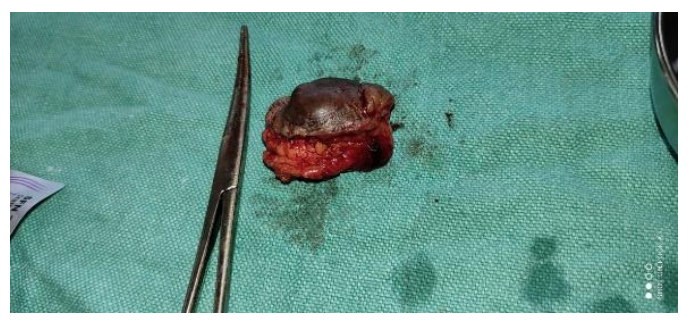

Figure 5: Scar endometrioma post excision.

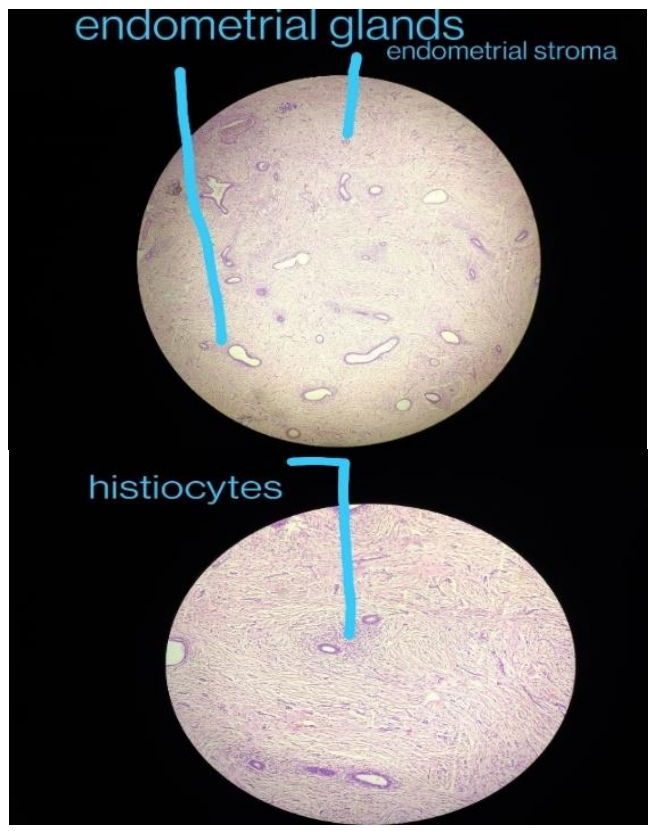

Figure 6: Histopathological slides confirming diagnosis of scar endometriosis.

\section{DISCUSSION}

Scar endometriosis is defined by the implantation or/and growth of endometrial tissue post-surgically at the incision site which is a very rare entity. The most common age group is 25-35 years and affects 10 to $15 \%$ of the fertile population. ${ }^{4}$ Caesarean section delivery is a very common cause and the frequency of scar endometriosis is increasing 
these days because of the increase in caesarean section rate.

The diagnosis of scar endometriosis is challenging and various differential diagnoses which may mimic the similar presentation include desmoid tumor, suture granuloma, fat necrosis, fibrosis, nodular fasciitis, hematomas, abscesses, and primary or metastatic malignancies. Scar endometriosis typically presents with cyclical changes in the severity of pain and increase size of endometrial implants during menstruation. Patients also usually complains of pain on palpation and an elevated unsightly hypertrophied scar or lump. However, in the largest reported series to date, only $20 \%$ of patients had these symptoms and were also reported in our case. ${ }^{5}$

A number of theories have been proposed which explains the origin of endometriotic tissue in the post-operative scars. These includes lymphatic or hematogenous dissemination, coelomic metaplasia and iatrogenic implantation of endometrial tissue during surgery are a few of them. ${ }^{6}$ Incidence is higher in hysterotomies and in caesarean sections which are performed prematurely or before the onset of labor pain. The decidua in early gestation have higher pluripotential properties and result in more cellular replication and also the immune tolerance to the fetal antigens mainly develops during later half of pregnancy.

The theories which have been postulated for pain in scar endometriosis includes neuronal invasion of endometriotic implant which subsequently derive sympathetic and sensory nerve supply which may undergo central sensitization leading to hyperexcitability of neurons causing persistent pain.

Also compared with eutopic endometrium, endometriotic implants are characterized by overproduction of local oestrogens, cytokines and prostaglandins which synergize the activities of each other, promoting implantation of ectopic endometrium and also pain associated with endometriosis. In addition, aromatase overactivity results in increased COX-2 expression favouring prostaglandin $\mathrm{E}$ production, which further upregulates oestrogen synthesis pathways. Management options therefore includes interventions that reduce ovarian oestrogen production reduce this synergistic process, thereby reducing or eliminating endometriosis-related pain. ${ }^{7}$

Ultrasonography, computed tomography, magnetic resonance imaging, fine needle aspiration cytology has been used for diagnosing this rare condition. ${ }^{8,9}$ The mass appears hypoechoic and heterogenous with internal echoes on ultrasonography. MRI is considered superior to CT scan mainly because of its high spatial resolution and better differentiation between the planes of muscle and sub cutaneous tissue. A recent case series study suggested that transabdominal sono-elastography may be beneficial in further delineating surfaces leading to more precise margin removal pre operatively. However, histopathological examination remains as the gold standard test for the diagnosis of scar endometriosis which shows the presence of endometrial glands, stroma, and hemosiderin pigment.

Medical management includes danazol, oral contraceptive pills, GNRH agonist but with limited success. Treatment of choice is wide local excision with clear margins along with reconstruction of damaged tissue to prevent recurrences. ${ }^{10}$ Synthetic mesh placement may be considered in larger lesions which lie deep to rectus sheath. Sclerotherapy with ethanol have been found to decrease the size of endometrioma but is not a definitive treatment modality. ${ }^{11}$

Authors in past have suggested that certain measures if taken can reduce the risk of development of scar endometriosis. The various measures includes avoidance of the same suture material which is used for uterine closure to close the layers of abdomen, washing and irrigating of the abdominal wound with normal saline to wash off the accidental endometrial tissue deposits at both ends of the scar and discarding the sponges which are used to clean endometrial cavity after removal of placenta and not using them for subsequent abdominal layers as it may lead to deposition of endometrial tissue in subsequent layers.

\section{CONCLUSION}

Scar endometriosis is a rare entity and may sometimes be difficult to diagnose. A high level of suspicion is required to diagnose a woman who present with pain at incisional site, most commonly presenting post pelvic surgery. A thorough history and physical examination should always be performed and every surgeon must consider this entity as their differential diagnosis. The measures described above should be incorporated into practice especially while training young obstetricians in order to reduce this surgical aftermath.

\section{ACKNOWLEDGMENTS}

Authors would like to thank Department of Obstetrics and Gynecology, Lokmanya Tilak Municipal Medical College and General hospital, Mumbai for their valuable support and co-operation of patients and their families admitted in this hospital.

\section{Funding: No funding sources \\ Conflict of interest: None declared \\ Ethical approval: Not required}

\section{REFERENCES}

1. Wolf GC, Singh KB. Cesarean scar endometriosis: a review. Obstet Gynecol Surv. 1989;44(2):89-95.

2. Padmanabhan LD, Mhaskar R, Mhaskar A. Scar endometriosis. J Obstet Gynaecol India. 2003;53:5961. 
3. Chatterjee SK. Scar endometriosis: a clinicopathological study of 17 cases. Obstet Gynecol. 1980;56:81-4.

4. Wolf Y, Haddad R, Werbin N, Skornick Y, Kaplan O. Endometriosis in abdominal scars: a diagnostic pitfall. Am Surg. 1996;62(12):1042-4.

5. Ding DC, Hsu S. Scar endometriosis at the site of cesarean section. Taiwan J Obstet Gynecol. 2006;45(3):247-9.

6. Sengul I, Sengul D, Kahyaoglu S, Kahyaoglu I. Incisional endometriosis: a report of 3 cases. Can J Surg. 2009;52(5):444-5.

7. Gupta P, Gupta S. Scar Endometriosis: a Case Report with Literature Review. Acta Med Iran. 2015;53(12):793-5.

8. Francica G, Giardiello C, Angelone G, Cristiano S, Finelli R, Tramontano G. Abdominal wall endometriomas near cesarean delivery scars: sonographic and color Doppler findings in a series of 12 patients. J Ultrasound Med. 2003;22:1041-7.

9. Gupta RK, Naran S. Aspiration cytodiagnosis of endometriosis in an abdominal scar after caesarean section. Acta Cytol. 1995;39:603-4.

10. Horton JD, Dezee KJ, Ahnfeldt EP, Wagner M. Abdominal wall endometriosis: a surgeon's perspective and review of 445 cases. Am J Surg. 2008;196:207-12.

11. Bozkurt M, Çil AS, Bozkurt DK. Intramuscular abdominal wall endometriosis treated by ultrasoundguided ethanol injection. Clin Med Res. 2014;12(3):160-5.

Cite this article as: Pathade $\mathrm{S}$, Panchbudhe $\mathrm{S}$, Deshmukh P, Nayak A. Scar endometriosis: an uncommon surgical aftermath. Int J Reprod Contracept Obstet Gynecol 2022;11:947-50. 